\title{
Incidental Early Gallbladder Carcinoma With Hepatocellular Carcinoma: A Rare Synchronous Double Primary Malignant Tumor
}

\author{
Mutlu Unver ${ }^{\mathrm{a}, \mathrm{c}}$, Safak Ozturk ${ }^{\mathrm{a}}$, Osman Bozbiyik ${ }^{\mathrm{a}}$, Varlik Erol ${ }^{\mathrm{a}}$, Eyup Kebapcia ${ }^{\mathrm{a}}$, Mustafa Olmez ${ }^{\mathrm{a}}$, \\ Umit Bayol $^{\mathrm{b}}$, Cengiz Aydina, Gokhan Akbulut ${ }^{\mathrm{a}}$
}

\begin{abstract}
We present a case of synchronous double primary tumor of gallbladder and liver. A 65-year-old male was admitted to the hospital complaining of abdominal discomfort and right upper abdominal pain. Abdominal computed tomography imaging showed acute cholecystitis with tiny gallbladder stones, a $2.2 \mathrm{~cm}$ size enhanced nodule between segment 7 and 8 and a $5.6 \mathrm{~cm}$ size hypodense lesion in the left lobe of the liver. The patient underwent a partial hepatectomy of the left lobe of the liver and a cholecystectomy. The result of histopathology confirmed the diagnosis as hepatocellular carcinoma (HCC) of the liver and gallbladder adenocarcinoma. The histopathologic examination of fine needle aspiration from the lesion between segment 7 and 8 also revealed an HCC and radiofrequency ablation was performed. The coexistence of an HCC and gallbladder adenocarcinoma is extremely rare. In conclusion, concomitant gallbladder carcinoma and HCC may occur with coexisting risk factors. Despite the more sophisticated diagnostic tools, it is not always possible to detect synchronus malignancies without histopathological examination. Radiofrequency ablation can be performed following surgery with low morbidity for preservation of liver function.
\end{abstract}

Keywords: Synchronous; Hepatocellular carcinoma; Incidental gallbladder carcinoma; Computed tomography; Fine needle aspiration

\section{Introduction}

Hepatocellular carcinoma (HCC) is a major public health problem and the sixth most common cancer worldwide with

\footnotetext{
Manuscript accepted for publication September 25, 2014

aDepartment of General Surgery, Tepecik Education and Research Hospital, 35100 Bornova, Turkey

bepartment of Pathology, Tepecik Education and Researching Hospital, 35100 Izmir, Turkey

${ }^{\mathrm{c} C}$ Corresponding Author: Mutlu Unver, Department of General Surgery, Tepecik Education and Research Hospital, 250 sok. No. 3/2 kat 7 daire 25 Manavkuyu, 35100 Izmir, Turkey. Email: mutluunver@gmail.com
}

doi: http://dx.doi.org/10.14740/jmc1961w about 500,000 new cases annually, representing the third largest cause of cancer-related death [1]. HCC has become the fastest growing cause of cancer-related death in men [2]. Most of the human HCC occurs after viral hepatitis (especially hepatitis B virus (HBV) or hepatitis $\mathrm{C}$ virus (HCV)) infections. In developing countries, $59 \%$ of liver cancers are attributable to HBV and, in developed countries, $23 \%$ of liver cancers are attributable to HBV [3]. In the preoperative setting, computed tomography (CT) is highly accurate in the staging of HCC, as the number of lesions, segmental anatomy, regional adenopathy, vascular tumour invasion and metastases can be detected easily. Gallbladder carcinoma is a less common malignancy than HCC with a female dominance. The main risk factors for gallbladder carcinoma are gallstones, anomalous pancreatic biliary-union, obesity and parity [4]. Synchronous double primary malignant neoplasms are a secondary malignancy occurring at the same time or within 6 months after the first malignancy. The coexistence of an HCC and gallbladder adenocarcinoma is extremely rare. The common embriyonic origin of gallbladder and liver may cause multiple cancers arising at different sites at the same time with well-known risk factors. This association, together with an analysis of the literature shows the increased incidence of gallstones in cirrhotic patients and the consequent greater surgical risk when undergoing subsequent cholecystectomy after liver resection. La Greca et al [5] suggested that cholecystectomy should be performed routinely during liver resection for HCC with or without cirrhosis, even for minor resections and when there are no evident signs of gallbladder disease.

\section{Case Report}

A 65-year-old male patient was admitted to our hospital complaining of abdominal discomfort and right upper abdominal pain for 3 months. The patient was chronic alcohol consumer and heavy smoker. The patient had chronic obstructive pulmonary disease (COPD) with no diagnosis and treatment. We began the treatment for COPD. There was no remarkable family history. On admission, vital signs (blood pressure, heart rate, respiration rate, and body temperature) were within normal limits. The patient was in good general health and had no significant weight loss. On physical examination, the abdomen was soft. The routine blood tests were normal. The platelet 

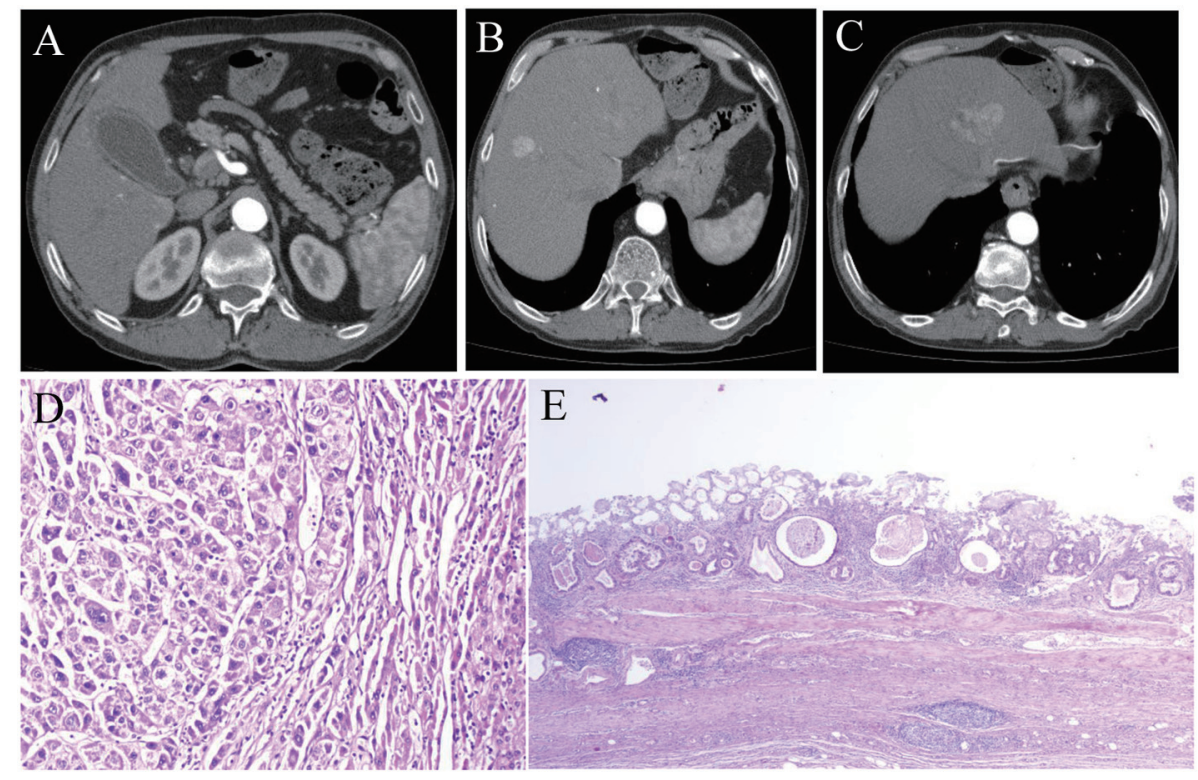

Figure 1. A 65-year-old male patient was admitted to our hospital complaining of abdominal discomfort and right upper abdominal pain. (A) Acute cholecystitis with tiny gallbladder stones and non-cirrhotic liver; (B) Nodule between segments 7 and 8 ; (C) Hypodense lesion in the left lobe of the liver; (D) Hepatocellular carcinoma consisting of solid, nodular tumor masses with pleomorphic hepatocyte like tumor cells; (E) Well differentiated adenocarcinoma of the gall bladder restricted to superficial muscularis propria.

count was $156,000 / \mathrm{mm}^{3}$. International normalized ratio was 1.56. Viral markers were hepatitis B surface antigen $(+)$, anti-HBs(-) and anti-hepatitis $\mathrm{C}$ virus(-). Tumor marker assays showed alpha-fetoprotein was $72 \mathrm{ng} / \mathrm{mL}$ (normal range (NR) $0-8.1 \mathrm{ng} / \mathrm{mL}$ ), carcinoembryonic antigen was $3 \mathrm{ng} / \mathrm{mL}$ (NR 0 - $5 \mathrm{ng} / \mathrm{mL}$ ), and carbohydrate antigen 19-9 was $11 \mathrm{U} / \mathrm{mL}$ (NR 0 - $37 \mathrm{U} / \mathrm{mL}$ ). The patient was in Child-Pugh A and MELD score was 12 . There were not esophageal varices in upper GI endoscopy. Abdominal CT imaging showed acute cholecystitis with tiny gallbladder stones (Fig. 1A), liver was non-cirrhotic, a 2.2 $\mathrm{cm}$ size enhanced nodule between segments 7 and 8 (Fig. 1B) and a $5.6 \mathrm{~cm}$ size hypodense lesion (Fig. 1C) in the left lobe of the liver. In the preoperative setting, the histopathologic examination of fine needle aspiration (FNA) from the lesion in the left lobe revealed an $\mathrm{HCC}$, so we prepared the patient with the diagnosis of HCC and acute cholecystitis caused by gallstones. At laparotomy, the gallbladder was slightly distended and showed wall thickening. The patient underwent surgical resection of the gallbladder and the left lobe of the liver 3 weeks after the diagnosis. Regional lymph nodes were dissected from pericholedochic area and intraoperative frozen section revealed reactive benign lymph nodes. The histopathologic examination after the operation revealed adenocarcinoma of gallbladder and HCC of liver (Fig. 1D). T stage of gallbladder cancer was T1a (Fig. 1E). The surgical margins were clean. On gross examination, the mass of the liver measured $7 \times 4 \times 3$ $\mathrm{cm}$. The patient recovered from the surgery with no complications. The patient was discharged on postoperative day 8 . The histopathologic examination of FNA from the lesion between segments 7 and 8 also revealed an HCC and radiofrequency ablation was performed 3 weeks after the surgery. Radiofrequency ablation (RFA) should be performed in the operating room but, the patient had COPD. For shortening the operation time, we decided to perform RFA after the surgery. Anti-viral treatment (lamivudin $100 \mathrm{mg} /$ day) was initiated and postoperative adjuvant chemotherapy (sorafenib $800 \mathrm{mg} /$ day) was planned. After a 6-month follow-up, he remains in good health with no signs of recurrence.

\section{Discussion}

Coexistence of an HCC and gallbladder adenocarcinoma is extremely rare. The occurrence rate of multiple primary malignancies is between $0.73 \%$ and $11.7 \%$ [6]. Multiple primary tumors could be synchronous or metachronous depending on the interval between their diagnosis. Synchronous tumors are second tumors occurring simultaneously or within 6 months after the first malignancy like as the present case. HCC is known to be pathogenically associated with liver cirrhosis, chronic hepatitis virus infection, and abuse of alcohol [7]. Gallbladders containing stones or infectious agents develop cancer as a result of recurrent trauma and chronic inflammation [8]. In the present case, gallbladder stones, abuse of alcohol and chronic HBV infection may have played an important role in the pathogenesis of gallbladder adenocarcinoma and HCC. Despite, hepatocellular and gallbladder carcinoma do not share common molecular pathways, the liver, gallbladder, bile ducts and pancreas have a common embryonic origin, so it is not surprising to have multiple cancers arising at different sites at the same time with well-known common risk factors, as they are expected to share similar spectrum of histologic types [4]. Simple cholecystectomy may be adequate treatment only for 
the earlier stages: Tis and T1a. All other stages, starting from T1b should be treated with lymphadenectomy and resection of at least 2 - $3 \mathrm{~cm}$ of liver parenchyma around the liver bed, provided that no residual microscopic cancer (R0) remains [9]. In the last decades, many local and regional techniques have been introduced, helping, together with surgery, to treat the HCC, like RFA. The benefits of this therapy include excellent local control comparable to surgical resection, low morbidity, repeatability for recurrence, preservation of liver function, and few complications [10]. In the present case we performed RFA with surgery for preservation of liver function and aimed to reduce morbidity and mortality. T stage of gallbladder carcinoma was T1a, so simple cholecystectomy was enough for adequate treatment.

To conclude, with well-known risk factors, the association of an HCC and gallbladder adenocarcinoma should be born in mind. This association, together with an analysis of the literature showing the increased incidence of gallstones in cirrhotic patients and the consequent greater surgical risk when undergoing subsequent cholecystectomy after liver resection, would suggest that cholecystectomy could be performed routinely during liver resection for $\mathrm{HCC}$ with or without cirrhosis, even for minor resections and when there are no evident signs of gallbladder disease in high risk patients with risk factors.

\section{Acknowledgement}

Authors also wish to thank Mrs. Crystal A Stang for editing English of the manuscript.

\section{References}

1. Parkin DM, Bray F, Ferlay J, Pisani P. Global cancer sta- tistics, 2002. CA Cancer J Clin. 2005;55(2):74-108.

2. El-Serag HB, Rudolph KL. Hepatocellular carcinoma: epidemiology and molecular carcinogenesis. Gastroenterology. 2007;132(7):2557-2576.

3. Garcia M, Jemal A, Ward EM, Center MM, Hao Y, Siegel RL, Thun MJ. Global cancer facts and figures 2007. Atlanta, GA: American Cancer Society.

4. Carriaga MT, Henson DE. Liver, gallbladder, extrahepatic bile ducts, and pancreas. Cancer. 1995;75(1 Suppl):171-190.

5. La Greca G, Barbagallo F, Rodolico M, Greco L, Randazzo V, Scuderi M, Russello D. Hepatocarcinoma, gallstone disease and gallbladder carcinoma: a case report of a rare incidental association. Chir Ital. 2004;56(4):571-574.

6. Demandante CG, Troyer DA, Miles TP. Multiple primary malignant neoplasms: case report and a comprehensive review of the literature. Am J Clin Oncol. 2003;26(1):7983.

7. Ohwada S, Yoshihiro O, Iwazaki S, Tanahashi Y, Sawada T, Takeyoshi I, Kawashima Y, et al. Double cancer in different hepatic lobes: hepatocellular and cholangiocellular carcinoma. Hepatogastroenterology. 1995;42(4):411414.

8. Kim JW, Han JW, Jung SY, Jung JP. Synchronous double primary malignant tumor of the gallbladder and liver: a case report. World J Surg Oncol. 2011;9:84.

9. Cavallaro A, Piccolo G, Panebianco V, Lo Menzo E, Berretta M, Zanghi A, Di Vita M, et al. Incidental gallbladder cancer during laparoscopic cholecystectomy: managing an unexpected finding. World J Gastroenterol. 2012;18(30):4019-4027.

10. Poon RT, Fan ST, Tsang FH, Wong J. Locoregional therapies for hepatocellular carcinoma: a critical review from the surgeon's perspective. Ann Surg. 2002;235(4):466486. 